\title{
Pyoderma gangrenosum: A case report of bilateral dorsal hand lesions and literature review of management
}

\author{
Donald S Mowlds MD MBA, Jeff J Kim MD, Patrick Murphy MD, Garrett A Wirth MD MS FACS
}

DS Mowlds, JJ Kim, P Murphy, GA Wirth. Pyoderma gangrenosum: A case report of bilateral dorsal hand lesions and literature review of management. Can J Plast Surg 2013;21(4):239-242.

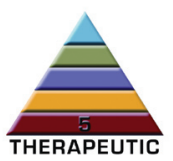

Pyoderma gangrenosum is a great masquerader in wound diagnosis and management. Frequently misdiagnosed as a necrotizing infection, the elusive nature of its etiology and pathogenesis has thwarted the establishment of a standardized management algorithm, leaving immunosuppressant therapies as the mainstay of treatment. The present report describes a 61-year-old woman presenting with temporally discrete bilateral dorsal hand lesions successfully managed with distinctive multimodality therapies. The initial lesion was managed under the auspices of a necrotizing process using a combination of hyperbaric oxygen therapy and skin grafting with a negative-pressure dressing, both individually demonstrated to be effective for prompt wound stabilization and coverage. A subsequent contralateral hand lesion was similarly managed as a necrotizing infection before a diagnosis of pyoderma gangrenosum was considered. Stabilization and eventual resolution was achieved using intravenous and topical steroids followed by hyperbaric oxygen therapy, again highlighting the benefits of multimodality therapy in the setting of pyoderma gangrenosum.

Key Words: Hyperbaric oxygen therapy; Immunosuppressants; Management; Negative pressure therapy; Pyoderma gangrenosum

Dyoderma gangrenosum (PG) is a rare but destructive cutaneous disease characterized by painful progressive ulceration and necrosis of the skin. Often difficult to distinguish from a necrotizing infection, it is most commonly located on the anterior surface of the lower leg. Presentation classically includes a necrotic centre expressing bloody or cloudy discharge and a rapidly advancing raised, red-purple border with an undermined edge. Hypothesized to be of immunological etiology, conservative treatment using prolonged high-dose systemic corticosteroids and other immunosuppressants, in addition to gentle local wound care, characterizes the mainstay of management. Failure of these traditional treatment modalities has prompted the use of more advanced interventions such as negative-pressure wound dressings and hyperbaric oxygen (HBO) therapy, with documented success (1-4). Here, we present an interesting case of temporally distinct PG lesions on the dorsal aspect of the hands, initially believed to be necrotizing infection, unintentionally but successfully treated with a combination of $\mathrm{HBO}$ therapy and negative-pressure vacuum-assisted closure. There is little published literature reporting a combined treatment approach for the management of PG. High clinical suspicion after the patient represented a few months later with a second lesion on the opposite hand over the analogous area finally led to the diagnosis of PG in our patient, which was subsequently treated successfully; this time, combining immunosuppressants with HBO therapy. In both treatments, rapid and stable wound closures were observed, with excellent patient outcome.

\section{CASE PRESENTATION}

A 61-year-old woman with a medical history that included hypertension, gastroesophageal reflux disease and hemochromatosis initially

\section{La pyodermite gangréneuse : rapport de cas de lésions bilatérales du dos des mains et analyse bibliographique de la prise en charge}

La pyodermite gangréneuse provoque souvent de faux diagnostics et de mauvaises prises en charge des plaies. Elle est souvent diagnostiquée à tort comme une infection nécrosante, et la nature insaisissable de son étiologie et de sa pathogenèse contrecarre la création d'un algorithme de prise en charge standardisé, laissant les immunosuppresseurs comme pivots du traitement. Le présent rapport décrit le cas d'une femme de 61 ans qui a consulté en raison de lésions bilatérales du dos des mains discrètes temporelles, prises en charge avec succès par des thérapies multimodales distinctives. La lésion initiale a été traitée sous les auspices d'un processus nécrosant faisant appel à un mélange de thérapie par oxygène hyperbare et de greffe de la peau accompagnée d'un pansement en pression négative, car il est démontré que chacun de ces traitements stabilise et couvre rapidement la plaie avec efficacité. Une lésion controlatérale subséquente a été également prise en charge comme s'il s'agissait d'une lésion nécrosante avant qu'on envisage un diagnostic de pyodermite gangréneuse. Les médecins ont réussi à stabiliser, puis à résoudre le problème à l'aide de stéroïdes intraveineux et topiques suivis d'une thérapie par oxygène hyperbare, faisant de nouveau ressortir les avantages de la thérapie multimodale en présence d'une pyodermite gangréneuse. presented with an abscess on the dorsal aspect of the right hand overlying the second metacarpalphalangeal joint. Suspecting an isolated necrotizing infection, local excision and wound care with normal saline wet-to-moist dressing changes were initiated by the hand surgeon. Plastic surgery was subsequently consulted and $0.25 \%$ acetic acid wet-to-moist dressing changes and $\mathrm{HBO}$ therapy was initiated. Four days later, the wound was covered with a full-thickness skin graft from the ipsilateral upper inner arm, which was secured using a negativepressure dressing (Wound VAC, KCI International, USA) for five days. Two months postoperatively, the skin graft was noted to have excellent take with only a small area of wound breakdown along the radial aspect at the wound margin (Figure 1). Rapid resolution was achieved with local wound care and daily outpatient $\mathrm{HBO}$ treatment (Figure 2). Antibiotics were started initially and discontinued because wound cultures repeatedly demonstrated no growth. Of note, during the initial workup, the patient was incidentally diagnosed with myelodysplastic syndrome via bone marrow biopsy performed subsequent to a macrocytic anemia and leukopenia discovered in her routine laboratory work.

Two months later, the patient represented with a new lesion, this time on the left hand (Figure 3). Suspecting a necrotizing infection, the hand surgery team proceeded with local debridement. At this time, wound cultures were negative and the patient lacked leukocytosis or fever. Despite aggressive intravenous antibiotic treatment with vancomycin, levofloxacin and clindamycin, the wound failed to improve, prompting the hand surgery team to perform a second debridement. The wound continued to progress, culminating in a $3 \mathrm{~cm} \times 3 \mathrm{~cm}$ ulcerated, necrotic, erythematous lesion overlying the dorsal aspect of her index finger metacarpal. At this point, plastic surgery was consulted.

Aesthetic and Plastic Surgery Institute, University of California, Irvine, California, USA

Correspondence: Dr Garrett A Wirth, Aesthetic and Plastic Surgery Institute, 200 South Manchester Avenue, Suite 650, Orange,

California 92868, USA. Telephone 714-456-3077, fax 714-456-2229, e-mail gwirth@uci.edu 


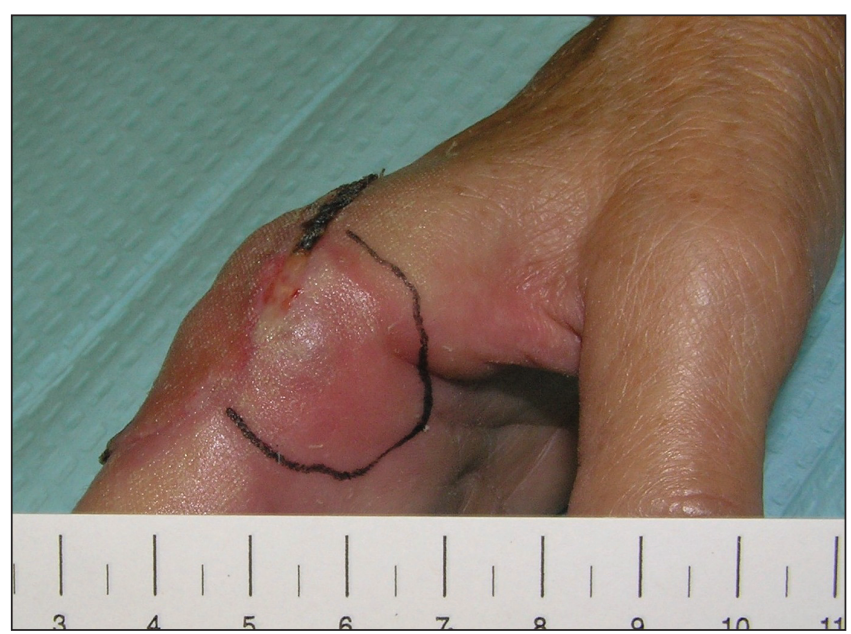

Figure 1) Full-thickness skin graft two months postoperatively on the dorsal aspect of the right hand overlying the second metcarpalphalangeal joint. It was secured using a negative-pressure dressing. Hyperbaric oxygen therapy resulted in excellent take after two months, with only a small area of wound breakdown along the radial aspect at the wound margin

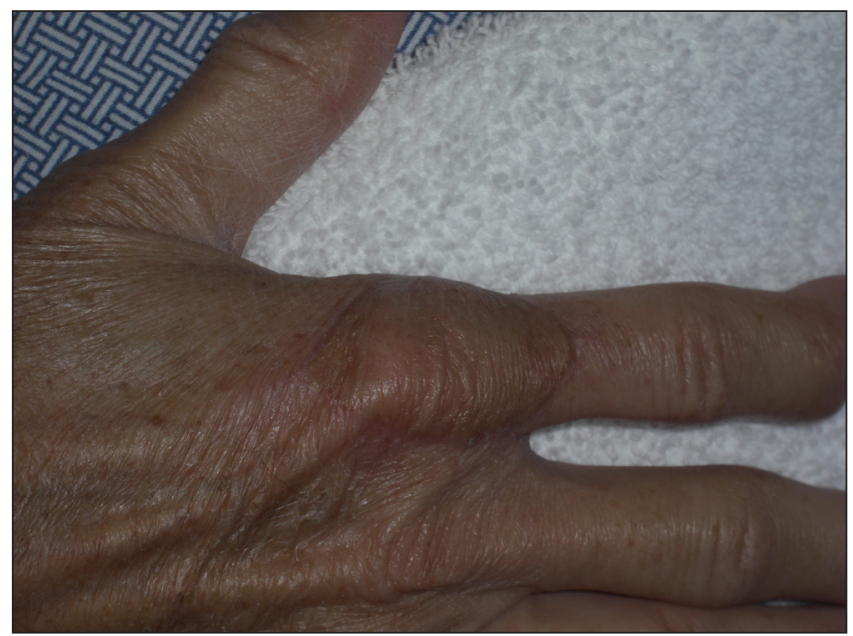

Figure 2) Rapid resolution of the right hand lesion, using continued local wound care and outpatient hyperbaric oxygen treatment

Owing to a lack of wound response to antibiotics and serial debridement, the clinical presentation of the remaining surrounding tissue and given the patient's newly diagnosed blood dyscrasia, a diagnosis of PG was considered by the plastic surgeon (GAW). Dermatopathology was subsequently consulted and lent support to the suspected diagnosis. The patient was immediately started on an intravenous steroid taper with topical tacrolimus ointment (Astellas Pharma, Japan) application to the wound perimeter and $\mathrm{HBO}$ therapy at $2.0 \mathrm{~atm}$ absolute twice per day for $90 \mathrm{~min}$. Approximately 20 treatments were delivered, at which time healthy granulation tissue was noted in the healing wound bed (Figure 4). At 10 weeks follow-up, complete, stable resolution of the wound was noted (Figures 5 and 6 ). There have been no documented recurrences in the 24 months following resolution (Figures 7 and 8).

\section{DISCUSSION}

PG warrants attention for several reasons. Not only does it closely resemble a necrotizing infection in terms of appearance, but also in its rapid progression of wound size (5). Diagnosis can be difficult because there are no pathognomonic findings on histological evaluation $(6,7)$. Furthermore, cultures are frequently misleading because, while PG in and of itself is not of microbial etiology, often superimposed infection is present in the wound $(8,9)$. Therefore, diagnosis remains dependent

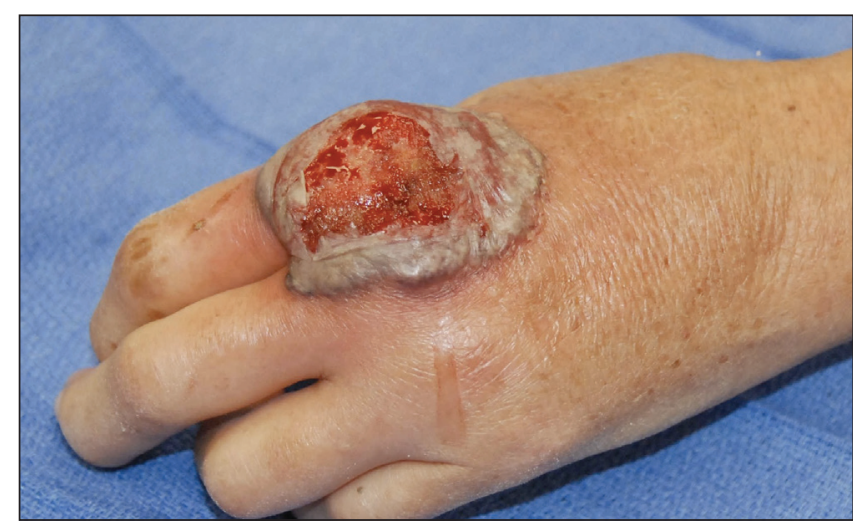

Figure 3) The second lesion, which appeared two months after resolution of the first, on the contralateral hand again on the dorsal aspect overlying the second metacarpalphalangeal joint

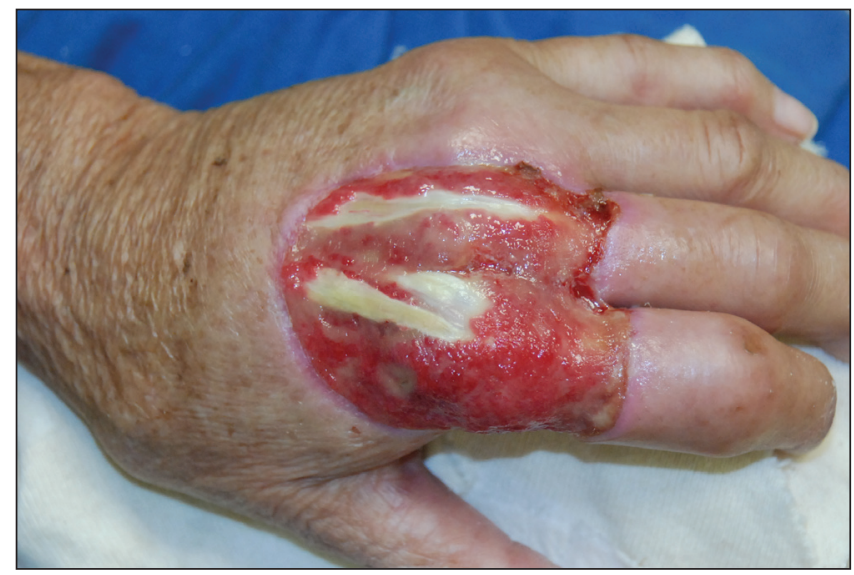

Figure 4) Left hand lesion showing healthy granulation tissue in the healing wound bed after the initiation of an intravenous steroid taper with topical tacrolimus ointment and approximately 20 outpatient hyperbaric oxygen therapy treatments at $2.0 \mathrm{~atm}$ for $90 \mathrm{~min}$, subsequent to diagnosis of pyoderma gangrenosum four weeks previously

on a high degree of clinical suspicion based on a history of systemic inflammatory disease or hematological malignancy, poor response to conventional treatment for a presumed infectious process and worsening after surgical intervention (10). Examining therapeutic options, it is clear why differentiation is important. Treatment of infectious processes involves systemic antimicrobials, which have no effect on PG, as well as debridement and excision of affected tissues, which can exacerbate the wound with worsening inflammation and ulceration through a phenomenon known as pathergy (11-13). Conventional treatment of PG involves gentle local wound care and systemic immunosuppression, which could prove disastrous in the setting of a necrotizing infection.

Because PG is a relatively rare occurrence, there is no definitive treatment algorithm. Early reports centred treatment on halting wound progression with systemic, intralesional and topical corticosteroids (14). Cyclosporine and other immunosuppressive agents, such as topical nitrogen mustard, certain antimicrobial agents, clofazimine and dapsone, which are typically used in the treatment of leprosy, evolved as adjunctive measures $(1,14,15)$. The other mainstay of treatment involved the avoidance of surgical intervention including debridement and skin grafting, which, as alluded to previously, may result in pathergy. Consequently, wounds were left to heal by secondary intention, which not only took a long time, but also frequently lead to 'cigarette paper thin' epidermal coverage when the wounds finally healed (14). Often, wounds were too large to ever completely heal and frequently exposed underlying structures, such as tendon or bone, which do not tolerate long periods of exposure (16). 


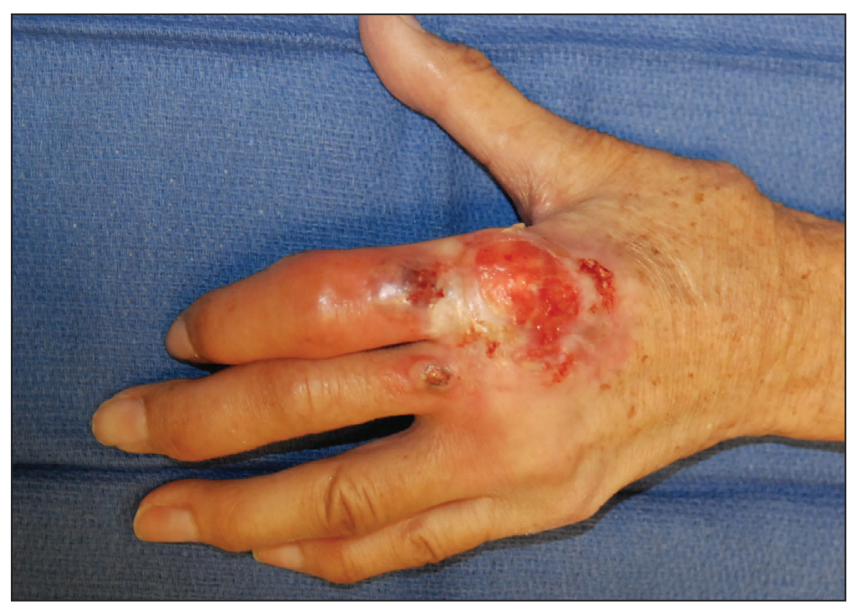

Figure 5) Left hand lesion after an intravenous steroid taper with local tacrolimus ointment application and approximately 20 outpatient hyperbaric oxygen therapy treatments leading to healthy granulation tissue in the healing wound bed and stable resolution at 10 weeks follow-up

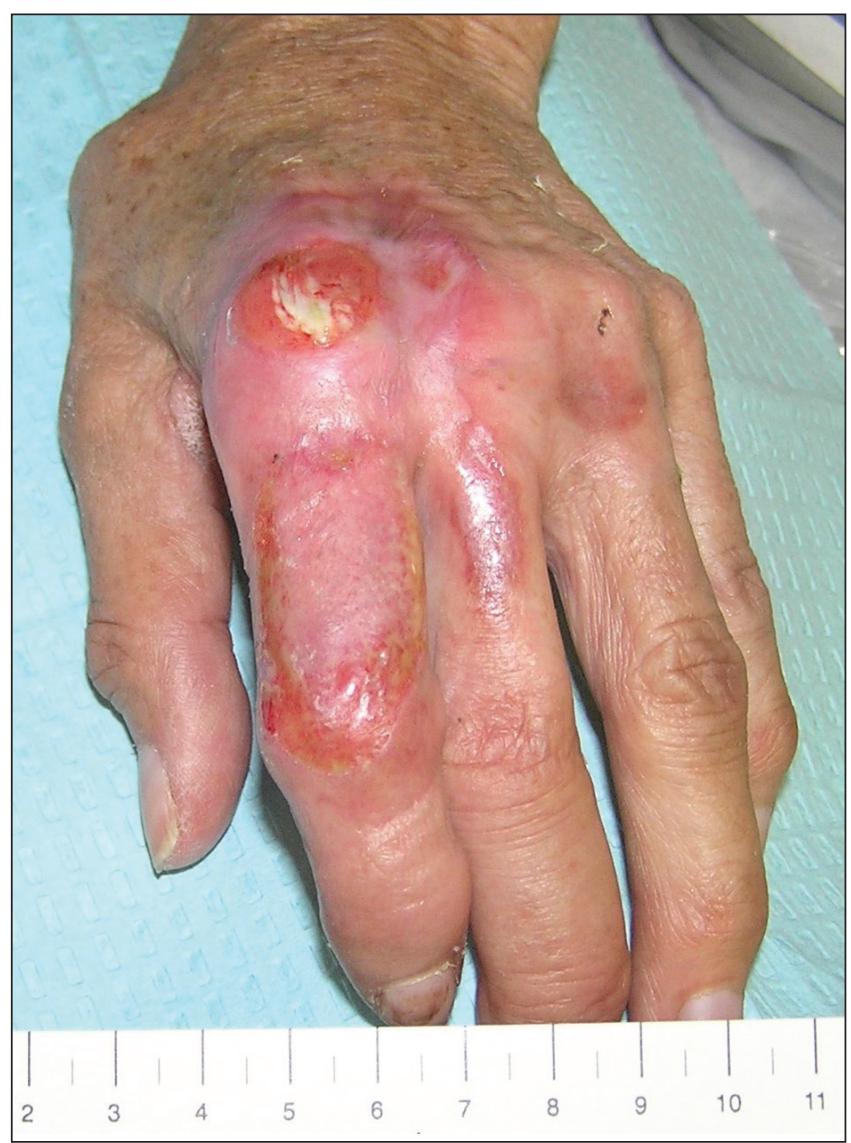

Figure 6) Left hand lesion after intravenous steroid taper with local tacrolimus ointment application and approximately 20 outpatient hyperbaric oxygen therapy treatments leading to healthy granulation tissue in the healing wound bed and stable resolution at 10 weeks follow-up

Prolonged wound care with unreliable epidermal coverage is obviously not ideal. Later efforts focused on stabilizing the wound to allow for more definitive coverage with a skin graft. Zakhireh et al (17) described stabilization of PG wounds with oral cyclosporine, allowing successful skin grafting after four to nine months (17). While this treatment algorithm allowed for eventual wound coverage, further studies sought a reduction in the time to achieve wound closure.

$\mathrm{HBO}$ therapy is gaining acceptance as an effective adjunctive measure in enhancing both the quality and timeline of wound healing

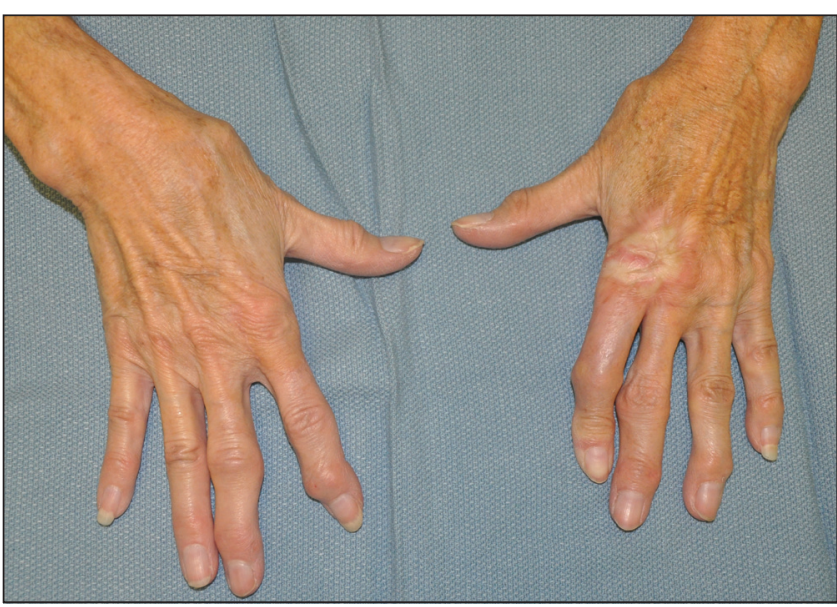

Figure 7) No documented recurrence 24 months post-treatment

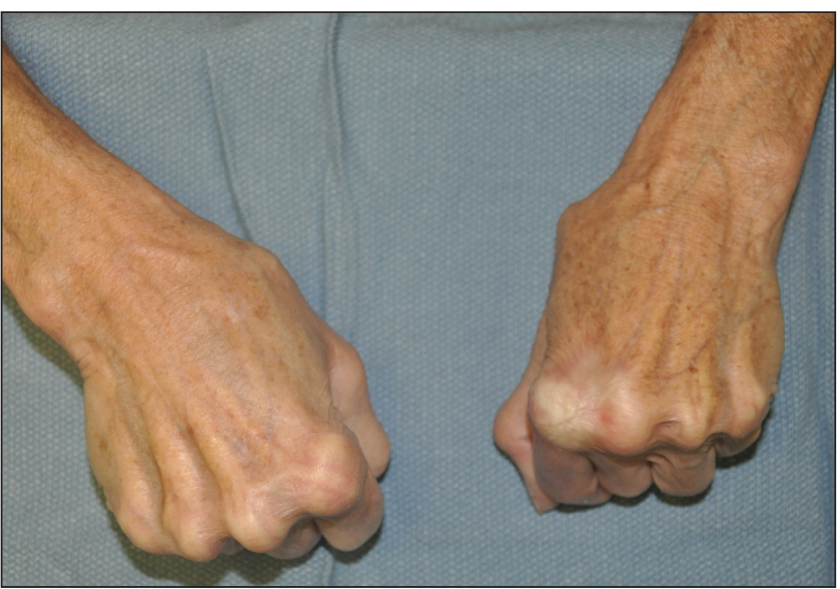

Figure 8) No documented recurrence 24 months post-treatment, with excellent maintenance of hand functionality

(1-4). It has been found to successfully stabilize progressive necrotizing wounds while also decreasing infection rates. The oxygen-rich environment provided by the therapy promotes angiogenesis and triggers a cascade of proliferative and inflammatory processes necessary for effective wound healing and closure, including nonspecific stimulation of collagen synthesis, granulation tissue formation and epithelial proliferation (4). There are several documented case studies highlighting the effectiveness of $\mathrm{HBO}$ therapy in treating PG ulcers and reducing pain associated with PG (18). Furthermore, $\mathrm{HBO}$ has been effective in PG ulcers refractory to other therapies. Mazokopakis et al (19) demonstrated safe and efficacious use of $\mathrm{HBO}$ therapy for lesions previously resistant to prednisolone, cyclosporine and etanercept. In a case reported by Davis et al (3), effective wound stabilization with HBO therapy in PG was used as a method of wound bed preparation before skin grafting. Four patients were successfully grafted after 28 to 82 days of $\mathrm{HBO}$ pretreatment, with no documented recurrences.

Negative-pressure dressings have been used to successfully treat problematic and refractory wounds of varying etiologies. Generally well tolerated and with few complications or contraindications, they are increasingly being used in a multitude of clinical settings, including the treatment of surgical wounds, traumatic wounds, diabetic foot ulcers and venous stasis wounds. The notion that cyclic application of subatmospheric pressure alters the cytoskeleton of the cells in the wound bed is well supported (20). The resultant cascade of intracellular signals has been shown to not only greatly increase the rate of granulation tissue formation, but also lower bacterial presence leading to accelerated wound healing (20). It has also been used to heal wounds by secondary intention or as a bolster type dressing to improve skin graft take, with Ghersi et al (21) demonstrating healing of PG wound by secondary intention specifically. The relevance of this modality to chronic 
wound management must not be understated because it not only provides an effective barrier to secondary infection, but also requires less frequent dressing changes and improves the local microcirculation, resulting in healthy granulation tissue formation (22).

These adjuvant therapies have individually been demonstrated to improve the timeline and quality of wound healing in the setting of PG. Combining them in a multimodality treatment regimen appears to be a logical extension of investigation. The current literature lends little support to such a strategy with the existence of only one previous report describing $\mathrm{HBO}$ therapy in concert with a negative pressure dressings and skin grafting. In this case report, Niezgoda et al (1) treated a lower extremity PG wound that failed four weeks of traditional treatment with oral steroids and local wound care. The ensuing treatment consisted of HBO therapy for three days to achieve wound stabilization and subsequent debridement with the application of a negative pressure dressing. On day 8 , the wound was covered with a split-thickness skin graft. HBO therapy and a negative-pressure dressing were continued postoperatively, which led to complete graft take and successful healing.

One other case report describing a combination of $\mathrm{HBO}$ and negative-pressure therapy - but without skin grafting - has been recently reported (16). In this case, wound breakdown after knee arthroplasty was ultimately diagnosed as PG after debridement, antibiotics and negative pressure treatment failed to achieve resolution. The wound stabilized after the initiation of prednisone and a gastrocnemius flap was used for coverage. The flap eventually broke down and reinitiation of negative-pressure treatment was unsuccessful until $\mathrm{HBO}$ therapy was started, allowing the wound to eventually contract and granulate.

Our patient underwent a multimodality treatment similar to that reported by Niezgoda et al (1), with the initiation of HBO therapy for wound stabilization before surgical intervention. However, unlike the experience of Niezgoda et al, debridement of the first lesion was foregone with direct procession to full thickness skin graft coverage and negative pressure dressing. Furthermore, immunosuppresants were not used in the initial wound stabilization because the diagnosis of PG had not been made at that time. Nevertheless, our combined experiences suggest that $\mathrm{HBO}$ with or without conventional medical treatment is

\section{REFERENCES}

1. Niezgoda JA, Cabigas EB, Allen HK, et al. Managing pyoderma gangrenosum: A synergistic approach combining surgical debridement, vacuum-assisted closure, and hyperbaric oxygen therapy. Plast Reconstr Surg 2006;117:24-8.

2. Wasserteil V, Bruce S, Sessoms SL, et al. Pyoderma gangrenosum treated with hyperbaric oxygen therapy. Int J Dermatol 1992;31:594.

3. Davis JC, Landeen JM, Levine RA. Pyoderma gangrenosum skin grafting after preparation with hyperbaric oxygen therapy. Plast Reconstr Surg 1987;79:200-7.

4. Heng MCY. Hyperbaric oxygen therapy for pyoderma gangrenosum. Aust NZ J Med 1984;14;618-21.

5. Bisarya K, Azzopardi A, Lye G, et al. Necrotizing fasciitis versus pyoderma gangrenosum: Securing the correct diagnosis! A case report and literature review. Open Access J Plast Surg 2011;11:218-27.

6. Bennett ML, Jackson MJ, Jorizzo JL, et al. Pyoderma gangrenosum: A comparison of typical and atypical forms with an emphasis on time to remission: Case review of 86 patients from 2 institutions. Medicine 2000;79:37-46.

7. Uwe W. Pyoderma gangrenosum - a review. Orphanet J Rare Dis 2007;2:19.

8. Callen JP. Pyoderma gangrenosum and related disorders. Adv Dermatol 1989;4:51-65.

9. Blitz NM, Rudikoff D. Pyoderma gangrenosum. Mount Sinai J Med 2001;68:287-97.

10. Ahronowitz I, Harp J, Shinkai K. Etiology and management of pyoderma gangrenosum: A comprehensive review. Am J Clin Dermatol 2012;13:191-211.

11. Kitagawa N, Ikeda M, Kodama H. Acute form of pyoderma gangrenosum introduced by traumatic injury. J Dermatol 1996;23:116-9.

12. Steadman UA, Brennan TE, Daman LA, Curry SL. Pyoderma gangrenosum following cesarean delivery. Obstet Gynecol 1998;91:834-6. effective in initially stabilizing the PG wound to the point at which it will tolerate invasive surgical procedures such as debridement and skin grafting. This is consistent with other descriptions of wound stabilization using $\mathrm{HBO}$ therapy, as discussed previously $(3,14,16,23)$. In terms of wound coverage and healing, had the diagnosis of PG been made, skin grafting would have been foregone, considering the relatively small size of the lesion and conventional wisdom against surgical intervention. Nevertheless, negative-pressure therapy in conjunction with continued $\mathrm{HBO}$ resulted in the rapid presentation of healthy, viable granulation tissue and excellent take of the grafted tissue, with continued stabilization and avoidance of postsurgical pathergy.

When our patient subsequently presented with a second lesion on the contralateral limb refractory to aggressive antibiotic treatment and surgical intervention, a diagnosis of PG was finally made. The patient underwent a combination of $\mathrm{HBO}$ therapy in concert with intravenous steroids and periwound tacrolimus application, which not only halted the wound progression, but also led to complete resolution and healing through secondary intention.

\section{SUMMARY}

While uncommon, PG is an important consideration in the setting of wound care, especially wounds recalcitrant to traditional treatment. Without accurate diagnosis and treatment, worsening of existing wounds is likely. Although there is no standardized treatment regimen, traditional treatment consisting of conservative wound care and medical management is likely to lead to prolonged healing times with inadequate soft tissue coverage. The present case demonstrates two effective multimodality approaches by direct comparison of similar wounds subjected to different therapies in the same patient, highlighting the manageability of surgical intervention and subsequent pathergy with proper wound stabilization. A combination of $\mathrm{HBO}$, topical tacrolimus and systemic steroids for wound stabilization before skin grafting and the application of negative-pressure dressings should, therefore, be considered in the multimodality approach to improving the timeline and quality of wound healing in the setting of PG.

DISCLOSURES: The authors have no financial disclosures or conflicts of interest to declare.

13. Finkel SI, Janowitz HD. Trauma and the pyoderma gangrenosum of inflammatory bowel disease. Gut 1981;22:410-2.

14. Wustrack KO, Zarem HA. Pyoderma gangrenosum: Recognition and management. Plast Reconstr Surg 1978;62:423-8.

15. Chow RKP, HO VC. Treatment of pyoderma gangrenosum. J Am Acad Dermatol 1996;34:1047-60.

16. Hill DS, O'Neill JK, Toms AM, et al. Pyoderma gangrenosum: A report of a rare complication after knee arthroplasty requiring muscle flap cover supplemented by negative pressure therapy and hyperbaric oxygen. Plast Reconstr Aesthet Surg 2011;64:1528-32.

17. Zakhireh, M, Rockwell WB, Fryer R. Stabilization of pyoderma gangrenosum ulcer with oral cyclosporine prior to skin grafting. Plast Reconstr Surg 2004;113:1417-20.

18. Tutrone WD, Green K, Weinberg JM, Caglar S, Clarke D. Pyoderma gangrenosum: Dermatologic application of hyperbaric oxygen therapy. J Drugs Dermatol 2007;6:1214-9.

19. Mazokopakis EE, Kofteridis DP, Pateromihelaki AT, Vytiniotis SD, Karastergiou PG. Improvement of ulcerative pyoderma gangrenosum with hyperbaric oxygen therapy. Dermatol Ther 2011;24:134-6.

20. Venturi ML, Attinger CE, Mesbahi AN, Hess CL, Graw KS. Mechanisms and clinical applications of the vacuum-assisted closure (VAC) device: A review. Am J Clin Dermatol 2005;6:185-94.

21. Ghersi MM, Ricotti C, Nousari CH, et al. Negative pressure dressing in the management of pyoderma gangrenosum ulcer. Arch Dermatol 2007143:1249-51.

22. Zutt M, Haas E, Kruger U, et al. Successful use of vacuum-assisted closure therapy for leg ulcers caused by occluding vasculopathy and inflammatory vascular diseases - a case series. Dermatology 2007;214:319-24.

23. Thomas EY, Crouch JA, Gustello J. Hyperbaric oxygen therapy for pyoderma gangrenosum. Arch Dermatol 1974;110:445-6. 